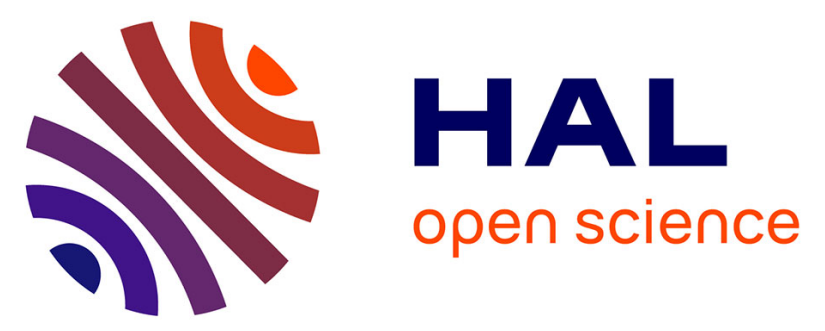

\title{
Solar Radius Determination from Sodism/Picard and HMI/SDO Observations of the Decrease of the Spectral Solar Radiance during the 2012 June Venus Transit
}

Alain Hauchecorne, Mustapha Meftah, Abdanour Irbah, Sebastien Couvidat, Rock Bush, Jean-François Hochedez

\section{To cite this version:}

Alain Hauchecorne, Mustapha Meftah, Abdanour Irbah, Sebastien Couvidat, Rock Bush, et al.. Solar Radius Determination from Sodism/Picard and HMI/SDO Observations of the Decrease of the Spectral Solar Radiance during the 2012 June Venus Transit. The Astrophysical Journal, 2014, 783 (2), pp.127. 10.1088/0004-637X/783/2/127 . hal-00952209

\section{HAL Id: hal-00952209 \\ https://hal.science/hal-00952209}

Submitted on 26 Feb 2014

HAL is a multi-disciplinary open access archive for the deposit and dissemination of scientific research documents, whether they are published or not. The documents may come from teaching and research institutions in France or abroad, or from public or private research centers.
L'archive ouverte pluridisciplinaire HAL, est destinée au dépôt et à la diffusion de documents scientifiques de niveau recherche, publiés ou non, émanant des établissements d'enseignement et de recherche français ou étrangers, des laboratoires publics ou privés. 


\title{
Solar radius determination from SODISM/PICARD and HMI/SDO observations of the decrease of the spectral solar radiance during the June 2012 Venus transit
}

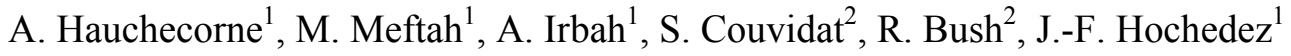 \\ 1 LATMOS, Université Versailles Saint-Quentin en Yvelines, UPMC, CNRS, F-78280 \\ Guyancourt, France, alain.hauchecorne@latmos.ipsl.fr \\ 2 W.W. Hansen Experimental Physics Laboratory, Stanford University, Stanford, CA 94305- \\ 4085, USA
}

\begin{abstract}
On 5 to 6 June, 2012 the transit of Venus provided a rare opportunity to determine the radius of the Sun using solar imagers observing a well-defined object, namely the planet and its atmosphere, occulting partially the Sun. A new method has been developed to estimate the solar radius during a planetary transit. It is based on the estimation of the spectral solar radiance decrease in a region around the contact between the planet and the Sun at the beginning of the ingress and at the end of the egress. The extrapolation to zero of the radiance decrease versus the Sun-to-Venus apparent angular distance allows estimating the solar radius at the time of $1^{\text {st }}$ and $4^{\text {th }}$ contacts. This method presents the advantage of being almost independent on the plate scale, the distortion, the refraction by the planetary atmosphere, and on the point-spread function of the imager. It has been applied to two space solar visible imagers, SODISM/PICARD and HMI/SDO. The found results are mutually consistent, despite their different error budgets: $959.85 " \pm 0.19 "(1 \sigma)$ for SODISM at $607.1 \mathrm{~nm}$ and $959.90 " \pm 0.06 "(1 \sigma)$ for HMI at $617.3 \mathrm{~nm}$.
\end{abstract}

Keywords: astrometry - Sun; Venus transit observations; fundamental parameters - solar radius

\section{Introduction}

In standard solar models the diameter of the Sun is assumed to be constant. The solar community has adopted a canonical radius value of 959.63 " at the end of the $19^{\text {th }}$ century (Auwers, 1891). However any change in solar diameter would indicate also a change in the energy radiated by the Sun. Solar models predict a link between the total luminosity and the photospheric radius (Spiegel and Weiss, 1980) that can be approximated to a linear relation:

$$
W=(D R / R /) /(D L / L)
$$

where $W$ is a dimensionless parameter, $R$ the photospheric radius and $L$ the total solar luminosity. There is a large uncertainty in the value of the parameter $W$ and the rare numerical modeling determinations differ both in sign and in magnitude (Fazel et al., 2008). An experimental determination would bring strong constraints to these models. In spite of significant progress in solar instruments and in the number of observations using different techniques, the question of the variability of the solar radius is still open and observational determinations give contradictory results concerning the relation between solar activity (and hence irradiance) on the one hand, and solar diameter on the other (Rozelot and Damiani, 2012). Laclare et al. (1996) and Morand et al. (2010), using solar astrolabe data respectively from 1975 to 1994 and from 1999 to 2006 at the Calern Observatory, found a mean value 
ranging from 959.40" to 959.48" and a 200 milliarcseconds (mas) variation that would be anti-correlated with the 11-year solar cycle. Egidi et al. (2006), analyzing data from the Solar Disk Sextant (SDS) onboard stratospheric balloons between 1992 and 1996, found also a 200 mas variation, from 959.50" to 959.70", anti-correlated with the solar activity. On the contrary, Bush et al. (2010), analyzing data from the Michelson Doppler Imager (MDI) aboard the Solar and Heliospheric Observatory (SOHO) from 1996 to 2010, found that any variation synchronous with the sunspot cycle must be smaller than 23 mas. There are also large discrepancies between the different determinations of the absolute solar radius, with estimated error bars much smaller than the observed differences. If we limit to modern published values, they range from 958.54" \pm 0.12 " (Sanchez et al., 1995) to 960.62" \pm 0.12 " (Wittmann, 2003) (for a review, see Kuhn et al., 2004; Thuillier et al., 2005; Djafer et al., 2008; Rozelot and Damiani, 2012). Solar radius measurements are difficult because they are affected by several parameters, among them the wavelength dependence, the plate scale, the point-spread function (PSF) and for ground observations atmospheric refraction and turbulence. Furthermore the Sun is not a solid body and the exact value of its radius is dependent on its definition, i.e. inflection point in the limb darkening function (LDF), edge of the limb, ...

One of the most promising techniques to determine the absolute solar radius is the use of transits of the Mercury and Venus planets. The solar radius is deduced from the measurement of the instant of external and internal contacts between the planetary disk and the Sun limb. The ephemerides of the planets and the Sun are known with a high accuracy, contributing to less than 5 mas to the solar radius error budget (4et al., 2013a). The transit method is not prone to errors in the plate scale of the telescope because the basic measurement is the time. However it may be affected by the point-spread function (PSF) of the optics and, for ground observations, by the atmospheric turbulence that makes the determination of the exact time of the transit more difficult.

Mercury transits takes place about 13 times per century (Shapiro, 1980) and more than 30 Mercury transits have been observed since the first observation in 1631 (Emilio et al., 2012).

These observations do not indicate any clear significant secular variation. This result does not confirm Ribes et al. (1987) who found a larger Sun by analyzing a 53-year record of regular observations of the solar diameter and sunspot positions during the seventeenth century. Venus transits are infrequent events. They occur on average 4 times every 243 years and only seven of them have been observed since the first one: 1639, 1761-69, 1874-82, 2004-12 (Sigismondi, 2013). Contrary to Mercury, Venus has an atmosphere producing refractive and absorptive effects that have to be taken into account in the determination of the Sun diameter. Transits of the internal planets Mercury and Venus over the Sun offer rare opportunities to determine the absolute value of the solar radius.

The exact determination of the solar radius using the time of the transit may be difficult if the quality of the images is degraded. This is especially the case for SODISM for which we observe a widening of the PSF (4et al., 2013b). In order to get around this problem, a new method has been developed. It is based on the estimation of the Sun radiance decrease just after the first contact and just before the fourth contact in an area surrounding the contact between Venus and the Sun and wide enough to encompass the PSF. As explained below, this method presents the advantage of being independent on the plate scale, the optical distortion and on the quality of the imager (PSF). It has been applied to images acquired by SODISM/PICARD and HMI/SDO during the 5-6 June 2012 Venus transit. PICARD is a CNES (Centre National d'Etudes Spatiales) microsatellite mission, launched on 15 June 2010, dedicated to the study of the Sun physics and Sun-Earth relations (Thuillier et al., 2006). Three instruments are embarked on board PICARD, two radiometers SOVAP (Solar 
Variability PICARD) and PREMOS (PREcision Monitor Sensor) measuring the Total Solar Irradiance (TSI) and the SOlar Diameter Imager and Surface Mapper (SODISM) providing $2048 \times 2048$ CCD images of the Sun at 5 wavelengths in the UV and visible spectrum (215.0, $393.37,535.7,607.1$ and $782.2 \mathrm{~nm})$.

The Helioseismic and Magnetic Imager (HMI) is an instrument designed to study oscillations and the magnetic field at the solar surface, or photosphere (Scherrer et al., 2012). HMI is one of three instruments on the Solar Dynamics Observatory (SDO); together, the suite of SDO instruments observes the Sun nearly continuously and takes two terabytes of data per day. HMI observes the full solar disk at $617.3 \mathrm{~nm}$ in the Fe I line and in the continuum near this line with a 4096 x 4096 CCD camera. HMI is a successor to the Michelson Doppler Imager (MDI) onboard the Solar and Heliospheric Observatory (SOHO).

In section 2, we describe the data used in the present work and we show the results obtained with SODISM and HMI. In section 3, we develop the method of analysis for the determination of the solar radius during the Venus transit. In section 4, we present the simulation made to validate the method, taking into account the clouds and the atmospheric refraction in the Venus atmosphere, and we show the results. In section 5, we conclude and we give some perspectives concerning the extension of this work to Mercury transits.

\section{Data description}

\section{SODISM/PICARD}

During the June 2012 Venus transit, a series of Sun images were acquired by SODISM. We chose to acquire images at $607.1 \mathrm{~nm}$ every minute during the ingress and again during the egress. Indeed, this spectral channel provides images in the photospheric continuum exhibiting the best PSF, and hence the sharper limb (Meftah et al., 2014b). Images obtained at 4 wavelengths $(393.37,535.7,607.1$ and $782.2 \mathrm{~nm}$ ) were taken alternatively during the central part of the transit but they are not used in this study. From the PICARD spacecraft orbit, the Venus transit started between 22:02 and 22:03 UTC on 5 June 2012 and ended between 04:43 and 04:44 on 6 June 2012. Level 0 CCD images are first corrected from the electronic offset, the dark signal and the flat-field. Accurate ephemerides for the position of Venus and Sun centers have been provided by IMCCE (Institut de Mécanique Céleste et de Calcul des Ephémérides http://www.imcce.fr/fr/ephemerides/). They are independent on any assumption on the solar radius. Ephemerides for the PICARD satellite are provided by CNES in the data files.

\section{$H M I / S D O$}

During the Venus transit the front camera of HMI took standard observables sequences with a 45 s cadence, while the side camera took true continuum images at 4 different polarizations. Here we only use side-camera data. Each image is separated by $3.75 \mathrm{~s}$ and is a snapshot with exposure duration of $0.125 \mathrm{~s}$, obtained at about $34.4 \mathrm{pm}$ from the central wavelength of the $\mathrm{Fe}$ I line at rest. The 4 polarizations taken, all linear, are (in Stokes-vector notation): I+Q, I-Q, $\mathrm{I}+\mathrm{U}$, and I-U. The change in polarization does not seem to have any impact on the measurement of the Venus radius. Therefore all of the 4 polarizations are used in this study, to take advantage of the higher acquisition rate. The images we work on are level 1 images that have been further treated. Basic level 1 data are raw HMI images that have been flat-fielded, have the CCD overscan rows and columns removed, and have been corrected for dark current and CCD bias. We then correct these images for cosmic-ray hits, bad CCD pixels, and the 
instrument distortion. On the resulting images, the plate scale is about 0.504" per pixel. Ephemerides of the Venus transit as seen from HMI/SDO are provided by the Goddard Space Flight Center. These ephemerides record the positions of the center of Venus across the solar disk at a cadence of $1 \mathrm{~s}$. Venus center coordinates are with respect to the Sun center.

\section{Data analysis}

\section{Estimation of the solar radiance decrease}

The method consists in the evaluation of the decrease of solar radiance during the beginning and the end of the transit. The last image before the first contact for the ingress and the first image after the fourth contact for the egress are used as reference. Table 2 summarizes the images used during the ingress and the egress and the corresponding reference images.

The procedure to estimate the radiance decrease is as follows:

- The difference between the actual image and the nearest reference image is computed (see Table 1 for images taken by SODISM and HMI).

- The radiance decrease is summed in an oval CCD area around the contact point with an extension that is large enough to cover the zone where the Venus disk occults the Sun taking into account the known width of the instrumental PSF. To test the validity of the results, we increase by steps of 1 pixel the size of the CCD area where the radiance decrease is averaged until a constant value is obtain. The CCD area is presented in color on Figure 1, the blue color representing the lowest decrease and the red color the highest.

- A possible change in the background signal, due for instance to a change in the CCD temperature, is monitored in an area where the Sun is not occulted (grey color in Figure 1) and the radiance decrease is corrected for this change. This area is an ellipse elongated along the solar limb in order to be at the same distance from the solar center than in the area where the radiance decrease is computed.

\section{Determination of the Sun radius}

If we consider two circles overlapping slightly each other (see for instance the SODISM image just after the first contact on left part of Figure 2), the common surface is in a first approximation proportional to the power $3 / 2$ of the overlapping distance along the line joining the Sun and Venus centers. This law assumes that the portion of Sun and Venus limbs intersecting each other can be approximated to portions of parabolas. A detailed demonstration of this law is given in Annex 1. If we assume also a uniform radiance on the solar disk, the solar flux decrease during the transit will be proportional to the occulted surface and its $2 / 3^{\text {rd }}$ power will be proportional to the overlapping distance. In the reality, there are some small deviations from this law due to the LDF and to the fact that the intersecting limbs have not a parabolic shape but a circular one. We assimilate Venus to a perfect disk with a diameter corresponding to the apparent altitude of the cloud top. The Venus solid radius is $6051.8 \mathrm{~km}$ and its apparent cloud top altitude has been fixed to $95 \mathrm{~km}$. The reason to choose this value is explained in section 4 .

\section{Validation of the method, results and error budget}


In order to validate the method, a simulation of the radiance decrease during the ingress and the egress has been performed. The Sun radiance has been computed as a function of the Sun center-to-Venus limb distance using the Hestroffer and Magnan (1998) analytic representation of the LDF. Venus has a thick atmosphere with a pressure of $9.10^{4} \mathrm{hPa}$ at the surface. However clouds cover permanently the planet and the sunlight crossing tangentially the Venus atmosphere during the transit cannot penetrate below the top of these clouds at around 80-90 km (Wilquet et al., 2009), about 0.1-1 hPa. In our simulation we fixed the altitude of the cloud top at $85 \mathrm{~km}$ with a total transmission above and no transmission below. Another important effect is the refraction of the Sun light in the Venus atmosphere that tends to reduce the apparent Sun radius at the beginning of the ingress and at the end of the egress. Mahieux et al. (2012) provide density and temperature profiles in the Venus upper atmosphere obtained with the SPICAV/SOIR high-resolution near infrared spectrometer on board the European Space Agency (ESA) Venus Express mission. It allowed us to estimate a mean profile of light deviation due to the atmospheric refraction. The result of the simulation is presented in Figure 3. The parabolic fit of the $2 / 3^{\text {rd }}$ power of the radiance decrease versus the Venus altitude at Sun edge is computed in the interval -60 to $+40 \mathrm{~km}$ It follows almost perfectly the simulated values in this altitude range and crosses the zero line at $95 \mathrm{~km}$. This altitude will be considered in our study as the apparent altitude of the cloud top. Above $40 \mathrm{~km}$ the simulated data deviate from the parabolic fit and the images taken in these conditions will not be used for the determination of the radius. This is the case for $3 \mathrm{HMI}$ images, 1 at the beginning of the ingress and 2 at the end of the outgress. The deviation from the parabolic fit of the radiance decrease at Venus altitudes greater than $40 \mathrm{~km}$ is mainly due to the refraction effect that depletes the solar limb. The limb shape at the extreme edge of the Sun may also play a role in this deviation. The refraction is in fact the dominant effect to determine the apparent altitude of the Venus cloud top. It starts to decrease the solar radiance much higher than the cloud top, up to $110 \mathrm{~km}$ and induces a $20-\mathrm{km}$ difference in the Venus altitude for a given radiance decrease (Figure 3). We estimate the uncertainty in the apparent zero crossing altitude to about $\pm 10 \mathrm{~km}$. It includes $\mathrm{a} \pm 5 \mathrm{~km}$ uncertainty in the cloud top altitude and $\mathrm{a} \pm 5$ $\mathrm{km}$ uncertainty due to the imperfect knowledge of the Venus atmospheric density profile. This induces $\mathrm{a} \pm 47$ mas uncertainty in the Sun radius determination.

\section{Results}

Figure 4 presents the $2 / 3^{\text {rd }}$ power of the radiance decrease versus the distance the Sun centerto-Venus limb distance for SODISM and HMI. A zoom is shown for HMI in Figure 5 for which more data are available with a small radiance decrease. A parabolic fit is applied to the data to take into account the small deviation from the linear relation. As explained in the previous section the $3 \mathrm{HMI}$ data points closest to the Sun edge are not included for the determination of the fit. The quality of the fit is much better for HMI due to more frequent images and a better signal to noise ratio. The results obtained from the zero crossing of the fits are:

$$
\begin{array}{ll}
\text { SODISM } & 959.851 ” \pm 0.170 ”(1 \sigma) \\
\text { HMI } & 959.897 ” \pm 0.008 ”(1 \sigma)
\end{array}
$$

The uncertainty given here represents only the quality of the fit and does not take into account other sources of uncertainty that are discussed in in the next sub-section.

The quality of the parabolic fit can be estimated from the residuals. Figure 6 presents the residuals for a linear fit to HMI data. These residuals follow well a parabolic shape, which justifies the use of a parabolic fit for the determination of the solar radius. A systematic 
difference is observed between ingress and outgress, corresponding to a 10-to-15 mas difference in solar radius. This difference may be due to a small uncertainty in the ephemeris and the timing of the image, or to a $2-$ to- $3 \mathrm{~km}$ difference in the apparent altitude of the top of Venus's clouds that is quite plausible.

\section{Error budget}

Table 2 provides the estimation of the global error budget at one standard deviation $(1 \sigma)$ assuming a quadratic sum of the errors due to the accuracy of the ephemeris and the quality of the linear fit and due to the uncertainty in refraction and absorption in the Venus atmosphere. The total error budget is found to be three times larger for SODISM (194 mas, rounded to 0.19") than for HMI (62 mas, rounded to 0.06"). The dominant term in the error budget is the quality of the fit for SODISM due to the rather poor signal to noise ratio and the low acquisition rate (1 image per minute). On the contrary the parabolic fit for HMI is of very high quality due to the higher acquisition rate (an image every 3.75 seconds) and a better signal to noise ratio. The dominant term is the uncertainty in Venus atmosphere parameters. The error budget includes the known statistical/random errors. We cannot exclude the existence of systematic unknown errors. Despite the relatively large budget error in SODISM determination, the radii values found using the two instruments differ only by 46 mas, indicating that the fit error for SODISM is perhaps overestimated.

\section{Conclusion and perspectives}

A new method has been developed to estimate the solar radius during the transit of a planet in front of the Sun. It is based on the estimation of the spectral solar radiance decrease in a region around the contact between the planet and the sun at the beginning of the ingress and at the end of the egress. The extrapolation to zero of the radiance decrease versus the Sun-toVenus distance allows estimating the solar radius at the time of $1^{\text {st }}$ and $4^{\text {th }}$ contacts. This method presents the advantage to be almost independent of the scale plate, the distortion and the PSF of the imager. It has been applied to the 2 space solar imagers SODISM/PICARD and HMI/SDO during the 5-6 June 2012 Venus transit. The results found, rounded to $959.85 " \pm$ $0.19 "(1 \sigma)$ for SODISM and $959.90 " \pm 0.06 "(1 \sigma)$ for HMI, are very similar despite the larger uncertainty in SODISM observations. The good agreement between SODISM and HMI results may be found unexpected due to the widening of the SODISM PSF. However, it may be explained by the fact that in our method we don't use the imagery but the integration of the CCD signal on an area broad enough to encompass the image of the occulted part of the Sun spread by the PSF. We are therefore quite insensitive to the degradation of the spatial resolution of the image, which can explain the good concordance between the values obtained from PICARD and SDO data. These values are also in a surprisingly good agreement with Meftah et al. (2014a) who found $959.86 " \pm 0.20$ " using the same $607.1 \mathrm{~nm}$ SODISM images during the 2012 Venus transit but with a totally different method based on the plate scale determination during the transit and the inflection point of the LDF. They are smaller than the value $960.12 " \pm 0.09$ " found by Emilio et al. (2012) using the MDI/SOHO observations at $676.8 \mathrm{~nm}$ during the 2003 and 2006 Mercury transits. No significant variation was observed over the 3 years between the two transits. In their analysis the plate scale was determined from the Mercury position in the solar image, in order to be insensitive to optical distortion, and the radius was computed from the position of the inflection point in the LDF. However we have to keep in mind that the Sun is not a solid body and does not have a well-defined surface. When comparing with other published results, we have to keep in mind that our method measures a solar radius that is not the one 
obtained by locating the inflection point in the LDF. The solar radius is therefore depending on the method used for its determination. In order to evaluate if the difference between our value and the one obtained by Emilio et al. (2012) is a true variation of the solar radius or if it is due to the method, we plan to apply our method to MDI data during 2003 and 2006 Mercury transits. The next Mercury transit will occur in 2016. We want to point out the great value of such observations during planetary transits for the determination of the solar radius and its possible changes. This is as strong argument to maintain in operation space solar imagers like HMI/SDO and SODISM/PICARD.

Acknowledgments PICARD is a mission supported by the Centre National d'Etudes Spatiales (CNES), the Centre National de la Recherche Scientifique (CNRS/INSU), the Belgian Space Policy (Belspo), the Swiss Space Office (SSO), and the European Space Agency (ESA). The HMI project is supported by NASA contract NAS5-02139. We thank the many colleagues who contributed to the PICARD mission as well as our colleagues from the SPICAV/Mars Express Team who provided useful information on Venus atmosphere.

\section{References}

Auwers, A., 1891, Astron. Nach., 128, 361.1891

Bush, R.I., Emilio, M., \& Kuhn, J.R., 2010, ApJ, 500, L195

Djafer, D., Thuillier, G., \& Sofia, S., 2008, ApJ, 676, 651

Egidi, A., Caccin, B., Sofia, S., et al., 2006, Sol. Phys., 235, 407

Emilio, M., Kuhn, J.R., Bush, R.I. , \& Scholl, I.F., 2012, ApJ, 750, 135

Fazel, Z., Rozelot, J.P., Lefebvre, S., et al., 2008, New Astronomy, 13, 65

Hestroffer,D., \& Magnan, C., 1998, Astronom. Astrophys., 333, 338

Laclare, F., Delmas, C., Coin, J.P., \& Irbah, A., 1996, Sol. Phys., 166, 211 et al., 1996

Mahieux, A., Vandaele, A.C., Robert, S., et al., 2012, J. Geophys. Res., 117, E07001

Meftah, M., Hauchecorne, A., Crepel, M., et al., 2014a, Sol. Phys. 289, 1

Meftah, M., Hochedez, J.-F., Irbah, A., et al., 2014b, Sol. Phys., 289, 1043

Morand, F., Delmas, C., Corbard, T., et al., 2010, C. R. Physique, 11, 660

Rozelot, J.-P., \& Damiani, C., 2012, The European Physical Journal H, 37, 709

Ribes, E., J.C. Ribe \& R. Barthalot, 1987, Nature, 326, 52

Sánchez, M., Parra, F., Soler, M., \& Soto, R., 1995, A\&A, 110, 3511995

Scherrer, P.H., Schou, J., Bush, R.I., et al., 2012, Solar Physics, 275, 207

Shapiro, I.I., 1980, Science, 208, 51

Sigismondi, C., 2013, proc. of the $4^{\text {th }}$ French-Chinese meeting on Solar Physics

Understanding Solar Activity: Advances and Challenges, 15 - 18 November, 2011 Nice, France, arXiv:1201.4011

Spiegel, E.A., \& N.O. Weiss, 1980, Nature, 287, 616 
Thuillier, G., Dewitte, S., \& Schmutz, W., 2006, Advances in Space Research, 38(8), 1792

Thuillier, G., Sofia, S., \& Haberreiter, M., 2005, Adv. Space Res., 35, 239

Wilquet, V., Federova, A., Montmessin, F., et al., 2009, J. Geophys. Res., 114, E00B42

Wittmann, A.D., 2003, Astron. Nachr., 324, 378 


\section{Annex: Relation between the Venus limb-to-Sun limb distance and the solar flux decrease}

We consider the beginning of the Venus transit just after the first contact.

Figure A1 shows the geometry of the occultation.

We assume a uniform radiance on the disk Sun. The flux decrease is then proportional to the area $S$ of the Sun portion occulted by Venus with

$$
S=A+B
$$

$A$ : area between the Sun limb and the $M B$ intersection line

$B$ : area between the Venus limb and the $M B$ intersection line

We want to find the relation between $S$ and the Sun limb-to-Venus limb distance $d$ along the line $P Q$ joining Sun and Venus centers where:

$$
d=a+b
$$

$a$ : distance from the Sun limb to the intersection line segment $M B$

$b$ : distance from the Venus limb to the intersection line segment $M B$

The areas $A$ and $B$ are expressed as:

$$
\begin{aligned}
& A=R_{S}^{2}\left(\Theta_{S^{-}} \cos \Theta_{S} \sin \Theta_{S}\right) \\
& B=R_{V}^{2}\left(\Theta_{V^{-}} \cos \Theta_{V} \sin \Theta_{V}\right)
\end{aligned}
$$

$R_{V}$ : Apparent Venus radius

$R_{S}$ : Apparent Sun radius

$\Theta_{V}$ : semi-angle of the intersection line segment seen from the Venus center

$\Theta_{S}$ : semi-angle of the intersection line segment seen from the Sun center

The distances $a$ and $b$ are expressed as:

$$
\begin{aligned}
& a=R_{S}\left(1-\cos \Theta_{S}\right) \\
& b=R_{V}\left(1-\cos \Theta_{V}\right)
\end{aligned}
$$

We consider now that we are just at the beginning of the transit with $\Theta_{V}$ and $\Theta_{S}<<1$. Expressions of the areas and the distances can approximated to the first term of their Taylor expansion:

$$
\begin{aligned}
& A \approx R_{S}\left[\Theta_{S^{-}}\left(1-\Theta_{S}{ }^{2} / 2\right)\left(\Theta_{S}-\Theta_{S}{ }^{3} / 6\right)\right] \approx R_{S}{ }^{2} \Theta_{S}^{3} / 3 \\
& B \approx R_{V}\left[\Theta_{V^{-}}\left(1-\Theta_{V}{ }^{2} / 2\right)\left(\Theta_{V}-\Theta_{V}^{3} / 6\right)\right] \approx R_{V}{ }^{2} \Theta_{V}^{3} / 3 \\
& a \approx R_{S}\left[1-\left(1-\Theta_{S}{ }^{2} / 2\right)\right] \approx R_{S}{ }^{2} \Theta_{S}{ }^{2} / 2 \\
& b \approx R_{V}\left[1-\left(1-\Theta_{V}{ }^{2} / 2\right)\right] \approx R_{V}{ }^{2} \Theta_{V}{ }^{2} / 2
\end{aligned}
$$


Using two expressions for the half-length of $M N$ segment:

$$
R_{S} \sin \Theta_{S}=R_{V} \sin \Theta_{V}
$$

approximated to:

$$
R_{S} \Theta_{S} \approx R_{V} \Theta_{V}
$$

the surface $S$ is expressed as:

$$
S=A+B \approx R_{S}{ }^{2} \Theta_{S}^{3} / 3+R_{V}{ }^{2} \Theta_{V}^{3} / 3=R_{V}{ }^{2} \Theta_{V}^{3}\left(1+R_{V} / R_{S}\right) / 3
$$

and the distance $d$ :

$$
d=a+b \approx R_{S}^{2} \Theta_{S}^{2} / 2+R_{V}^{2} \Theta_{V}^{2} / 2=R_{V} \Theta_{V}^{2}\left(1+R_{V} / R_{S}\right) / 2
$$

Finally, the relation between $d$ and $S$ is expressed as:

$$
d \approx\left(3^{2 / 3} / 2\right) R_{V}^{-4 / 3}\left(1+R_{V} / R_{S}\right)^{1 / 3} S^{2 / 3}
$$

This justifies the use of a $2 / 3^{\text {rd }}$ power law for the relation between the Venus limb-to-Sun limb distance and the solar flux decrease assuming a uniform radiance on the Sun disk. 


\section{Tables}

Table 1. Images used for the estimation of radiance decrease during the ingress and the egress

\begin{tabular}{|l|l|l|}
\hline & $\begin{array}{l}\text { Date and UTC time } \\
\text { of the image }\end{array}$ & $\begin{array}{l}\text { Date and UTC time of } \\
\text { the reference image }\end{array}$ \\
\hline $\begin{array}{l}\text { SODISM } \\
\text { ingress }\end{array}$ & 5 June, 22:03 & 5 June, 22:02 \\
\hline “ & 5 June, 22:04 & 5 June, 22:02 \\
\hline “ & 5 June, 22:05 & 5 June, 22:02 \\
\hline SODISM egress & 6 June, 04:42 & 6 June, 04:44 \\
\hline & 6 June 04:43 & 6 June, 04:44 \\
\hline HMI ingress & 5 June, 22:08:25:22 & 5 June, 22:08:13:97 \\
\hline & 5 June, 22:08:28:97 & 5 June, 22:08:13:97 \\
\hline & Every 3:75 seconds & 5 June, 22:08:13:97 \\
\hline & 5 June, 22:08:55:22 & 5 June, 22:08:13:97 \\
\hline HMI egress & 6 June, 04:34:43:98 & 6 June, 04:35:28:97 \\
\hline & 6 June, 04:34:47:72 & 6 June, 04:35:28:97 \\
\hline Every 3:75 seconds & 6 June, 04:35:28:97 \\
\hline 6 June, 04:35:13:97 & 6 June, 04:35:28:97 \\
\hline
\end{tabular}

Table 2. Error budget for the determination of the solar radius

\begin{tabular}{|c|c|c|}
\hline & SODISM/PICARD & HMI/SDO \\
\hline Ephemeris & 5 mas & 5 mas \\
\hline $\begin{array}{l}\text { Spacecraft position/date } \\
\text { (probably pessimistic) }\end{array}$ & 80 mas & 40 mas \\
\hline Parabolic fit & $170 \mathrm{mas}$ & 8 mas \\
\hline Venus atmosphere & 47 mas & 47 mas \\
\hline Quadratic sum & $194 \operatorname{mas}(1 \sigma)$ & $62 \operatorname{mas}(1 \sigma)$ \\
\hline
\end{tabular}




\section{Figures}

Figure 1. Estimation of the radiance decrease in the SODISM image on 5 June, 22:05 UTC. The colored zone represents the area where the radiance decrease is averaged and the grey zone represents the area used for the determination of the background evolution.

Figure 2. Part of SODISM images around the zone of Venus transit (left) at the beginning of the ingress and (right) when Venus is fully in front of the sun.

Figure 3. Simulation of the $2 / 3^{\text {rd }}$ power of the solar radiance decrease versus the Venus altitude at Sun edge. The LDF is assumed to follow the analytic representation given by Hestroffer and Magnan (1998). In this simulation the refraction in the Venus atmosphere is taken into account as described in section 3 and the Venus cloud top is fixed at $85 \mathrm{~km}$.

Figure 4. Two-third power of the solar radiance decrease as a function of the distance between the Sun center and the Venus limb (see text): HMI ingress blue dots, HMI egress red dots, HMI linear fit red line, SODISM ingress blue stars, SODISM egress red stars, SODISM linear fit black line. Radiance decreases obtained by HMI and SODISM are in arbitrary.

Figure 5. Two-third power of the solar radiance decrease as a function of the distance between the Sun center and the Venus limb (see text): HMI ingress blue dots, HMI egress red dots, HMI linear fit black line. Radiance decrease is in arbitrary.

Figure 6. Residual from the linear fit to the $2 / 3^{\text {rd }}$ power of the solar radiance decrease versus the Sun center-to-Venus limb distance for HMI data. The solid line shows the parabolic fit to these residuals. The 3 last points are excluded to compute the fit as explained in the text.

Figure A1. Geometry of the transit. 


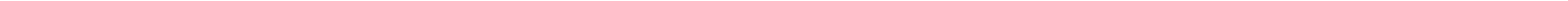




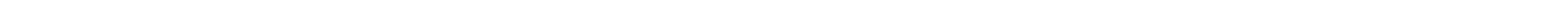




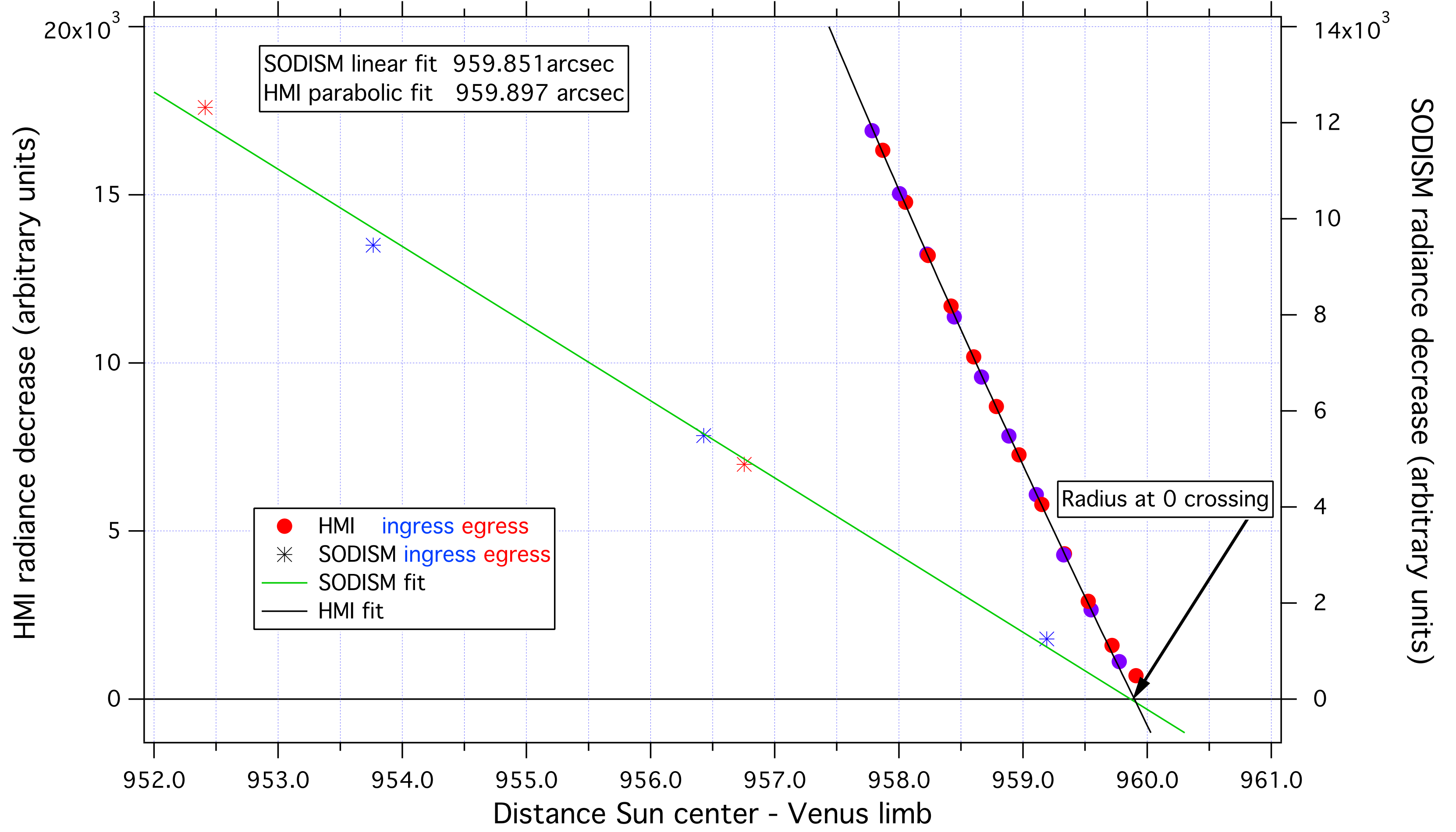




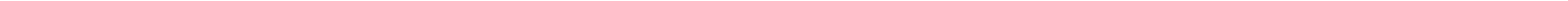




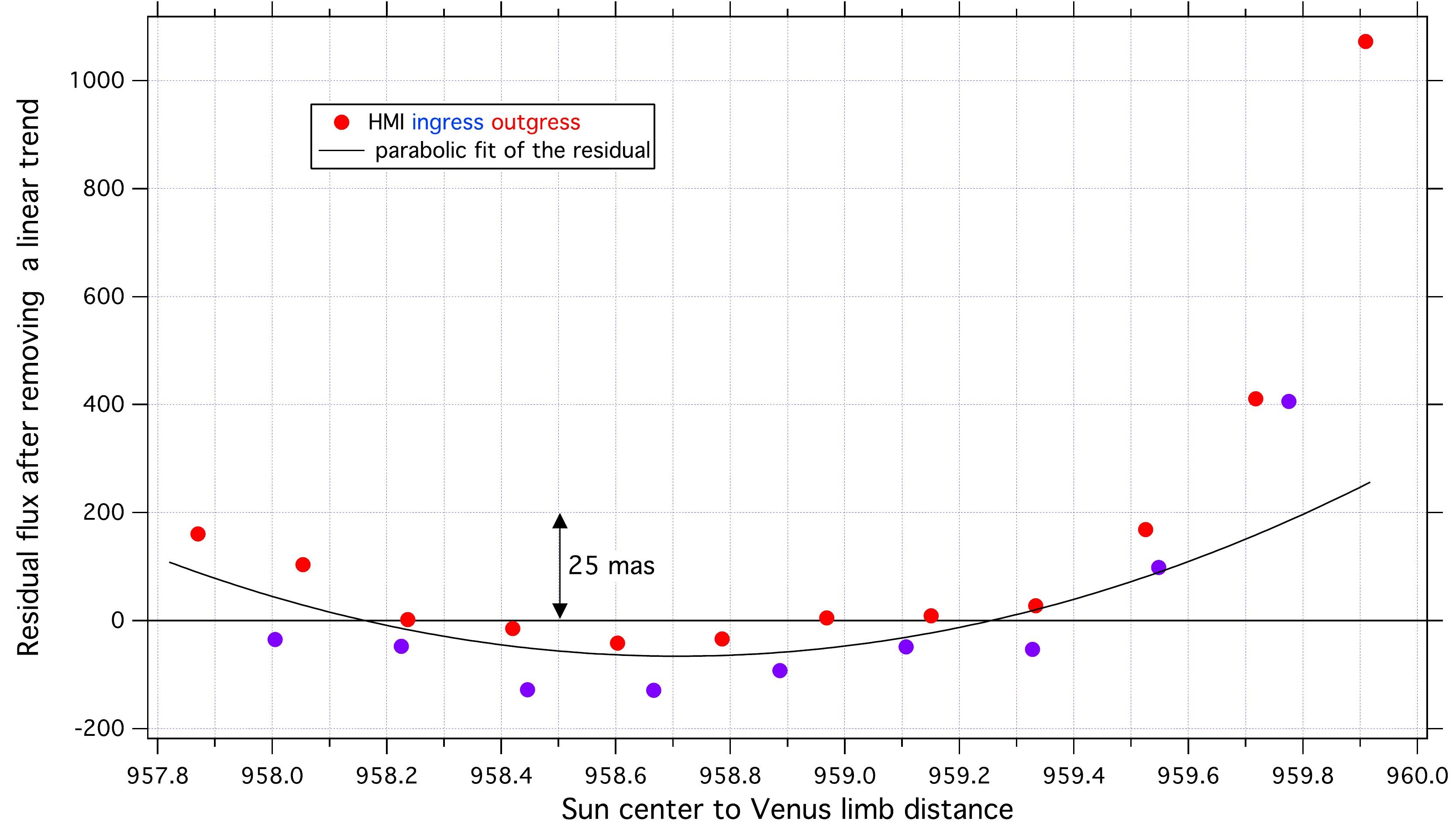




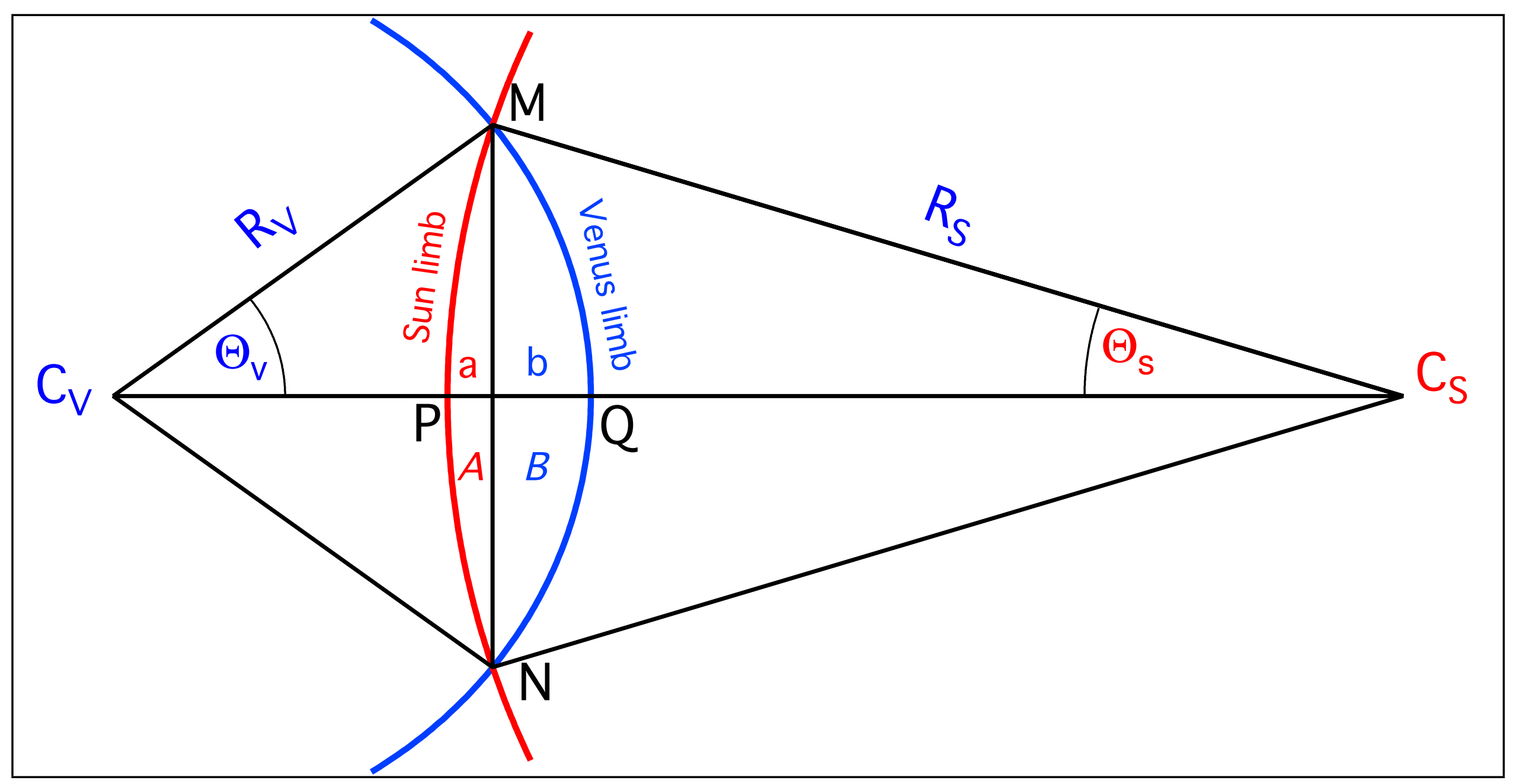

